\title{
The significance of Akt/NF- $k b$ signaling pathway in the posterior cataract animal model
}

\author{
Shao DW ${ }^{1}$, Zhu $\mathrm{XQ}^{1}$, Huo L ${ }^{1}$, Sun $\mathrm{W}^{1}$, Pan $\mathrm{P}^{1}$, Chen $\mathrm{W}^{2}$, Wang $\mathrm{H}^{2}$, Liu $\mathrm{B}^{2}$ \\ Department of Ophthalmology, Air Force Aviation Medicine Research Institute Affiliation Hospital, \\ Beijing, China. shaodewang2016@sina.com
}

\section{ABSTRACT}

OBJECTIVE: To establish SD rat posterior capsular opacification (posterior capsular opacification- PCO) animal model, and to detect the expression of Akt/NF-kb signaling pathway in the PCO model.

METHODS: 30 healthy SD rats were randomly divided into control group (0d) and the experimental groups $(7 \mathrm{~d}$ and $14 \mathrm{~d}$ ), there were 10 rats at all time points. All rats (right eye) were treated with the lens capsule, and the inflammatory reaction of the anterior segment of the eye and the occurrence of PCO at different time points were observed under the microscope. The TGF- $\beta$ concentration of humor aquosus was measured at the different time points by ELISA method. Eyeballs were removed after the rats were killed. RT-PCR method was used to detect the gene expression levels of Akt and NF-kb and Westen Blot method to detect the protein expression of Akt, p-Akt, NF-kb and p-NF-kb.

RESULTS: TGF- $\beta$ concentration, Akt and NF-kb gene expression, and Akt, p-Akt, NF-kb and p-NF-kb protein expression in humor aquosus, increased with the time and the time-dependence was significant.

CONCLUSION: Akt/NF-kb signaling pathway may be closely related to the occurrence and development of PCO, which may be related to the role of protein phosphorylation (Fig. 5, Ref. 20). Text in PDF www.elis.sk. KEY WORDS: Akt, NF-kb, TGF- $\beta$, PCO.

\section{Introduction}

Posterior capsule opacification (PCO), also known as posterior capsule opacification is the most frequent complication of cataract surgery. After cataract surgery, the incidence of $1 \sim 5$ PCO D was $50 \% \sim 11 \%$, and the incidence rate was $100 \%$ (1). The study showed that the proliferation, migration and epithelial mesenchymal transition (EMT) of lens epithelial cells (LEC) after cataract surgery were the main reasons for the formation of PCO (2). Transforming growth factor (TGF) can induce the occurrence of EMT. Previous studies have shown that the highest content of TGF- beta in the water can effectively induce the occurrence of EMT LEC, which is essential for the occurrence of PCO (3), This method has become a cell model for studying PCO (4). Studies have shown that TGF plays an important role in the induction of EMT in retinal pigment epithelial cells $(5,6)$. But so far, the research on the upstream channel of TGF in PCO is relatively limited. In this study, we used RT-PCR, Elisa and Westen Blot methods to study the roles of Akt/NF- $\mathrm{kb}$ pathway in PCO pathogenesis.

${ }^{1}$ Department of Ophthalmology, Air Force Aviation Medicine Research Institute Affiliation Hospital, Beijing, China, and ${ }^{2}$ Department of Ophthalmology, Air Force General Hospital, Beijing, China

Address for correspondence: D.W. Shao, Department of Ophthalmology, Air Force Aviation Medicine Research Institute Affiliation Hospital, Beijing, 100089, China.

\section{Material and methods}

\section{Main reagents and instruments}

Department of ophthalmology operation microscope (Suzhou six six visual Polytron Technologies Inc), Surgical instruments in Department of Ophthalmology(Suzhou medical instrument factory), Compound Tropicamide Eye Drops (Shenyang Shengyuan Pharmaceutical Co. Ltd.), Oxybuprocaine Hydrochloride Eye Drops (Santen Pharmaceutical Co., China), Tobramycin and Dexamethasone Eye Ointment (American Alcon Corporation), Atropine Sulfate Eye Ointment (Shanghai General Pharmaceutical Limited by Share Ltd), Hyaluronic acid sodium salt (The victory, Shandong bauschlomb Freda), Anti Sheep anti mouse, immune tissue chemical Kit (Zhongshan Company, Beijing), Anti Akt, anti p-Akt, anti NF-kb antibody (Abochorage Shanghai Trading Co., Ltd.), Rat TGF- beta ELISA Kit (West Tang Biotechnology Co., Ltd., Shanghai).

\section{Experimental animals and groups}

30 healthy female adult clean grade SD rats, body weight 240 $\sim 260 \mathrm{~g}$, under the microscope observation, exclude the anterior segment lesions. The rats were divived into control group and two experimental groups. All rats were under general anesthesia and lens was removed from the right. In the control group it was performed immediately $(0 \mathrm{~d})$, and in the experimental groups 7 days and 14 days after operation. 
423-426

\section{Methods}

Operation method

The operation was performed by the same operator with the improved rat lens capsule. Measuring the body weight $30 \mathrm{~min}$ preoperatively, using $40 \mathrm{~g} / \mathrm{L}$ hydrate $(0.8 \mathrm{ml}$ per $100 \mathrm{~g})$ intraperitoneal injection, general anesthesia 3 times after mydriasis, the rats were fixed on the operating table, Conjunctival sac instillation of 40 g/L Oxybuprocaine Hydrochloride Eye Drops 3 times, each time 1 drops; Saline eye surgery, compound iodine disinfection eye surgery in a clear corneal incision, injecting viscoelastics, keeping anterior chamber; Along the puncture mouth to expand the incision about 180 degrees, Circular capsulorhexis, about $5 \mathrm{~mm}$ in diameter, water separation to a nuclear free bag, compression method lens delivery and cortex, incision was sutured to the water dense and the anterior chamber was formed. The conjunctival sac with TobraDex and atropine. Postoperative daily observation in rats with systemic and ocular conditions, PRED Forte eyedrops conjunctival sac eye drops, 3 times a day, 1 drop each time, at night Tu Dian of TobraDex and atropine, a total of 7 days. Respectively on postoperative days 0,7 and 14 general anesthesia under the microscope observation of anterior segment inflammation and the capsule, fully dilated before each observation and photographic system recorded images.

\section{Materials and specimen handling}

On days 0,7 and 14 after operation the water in the anterior chamber was extracted under general anesthesia, and kept at -80 ${ }^{\circ} \mathrm{C}$ until the measurements were done. Each group was randomly sacrificed 5 only posterior capsular tissues into the $15 \mathrm{~mL}$ EP tube, liquid nitrogen preservation. In each of the remaining five rats eye surgery was performed, lens was fixed in $40 \mathrm{~g} / \mathrm{L}$ poly formalin, paraffin embeded sliced, patched, hematoxylin eosin stained, neutral gum mounting and observed with optical microscope.

\section{HE staining}

The rats were sacrificed after the removal of posterior capsular tissue that was fixed with par formaldehyde, embedded, sliced; HE stained and observed under light microscope.

\section{Elisa method}

The expression of TGF-beta in water at different time points was collected on days 0,7 and 14 of water, each hole was added to the $100 \mathrm{~L}$ test sample (activated), and the reaction plate was fully mixed at $37^{\circ} \mathrm{C}$ for $40 \mathrm{~min}$; Washes the reaction plate and washed thoroughly $4 \sim 6$ times and on filter paper dry and India, each hole to join the distilled water and the working fluid in the first antibody, $50 \mathrm{mu}$ L (blank except), fully mixed evenly rear 37 DEG C for 20 min; plate washer ibid. Each hole enzyme marked antibody working fluid $100 \mathrm{~g} / 1,37 \mathrm{DEG}$ C for $10 \mathrm{~min}$; plate washer ibid. Each hole adding substrate solution 100 g/l, set 37 degrees and a dark reaction for $15 \mathrm{~min}$; Each hole was added to $100 \mathrm{~L}$ liquid mixture, and the absorbance value was measured at $30 \mathrm{~min}$ using the enzyme marker at $450 \mathrm{~nm}$.

\section{RT-PCR test}

The total RNA was extracted from the tissues of each group, and the template for reverse transcription was cDNA. Primer sequence as follow, Akt: F: 5'-GGACAACCGCCATCCAGACT-3'

\section{R: 5'-GCCAGGGACACCTCCATCTC-3' \\ NF-kb: F: 5'-AATTGCCCCGGCAT-3' \\ R: 5'-TCCCGTAACCGCGTA-3' \\ $\beta$-actin: F: 5'-GCCTCGCTGTCCACCTTCCA-3' \\ R: 5'-CACCTTCACCGTTCCAGTTT-3'}

Using cDNA as a template, using GAPDH as reference, according to the SYBR Primix Ex Taq II (TaKaRa Kit) was set to 3 holes. Reaction conditions: 95 30s; 95 5s; 60 C 31s, 40 cycles. The $2^{-\Delta \Delta \mathrm{Ct}}$ value indicates the expression of mRNA.

\section{Western Blot test}

From that of the control group and experimental group lens posterior capsular tissue, adding proper amount of lysate and cracking after $10000 \mathrm{R} / \mathrm{min}$ centrifugation for $5 \mathrm{~min}$, the supernatant was full. Add up 5 folds sample buffer, cooking $5 \mathrm{~min}$ at $100{ }^{\circ} \mathrm{C}$, fully mixed. SDS-PAGE gel configuration, sample, electrophoresis, transfer film. $50 \mathrm{~g} / \mathrm{L}$ skimmed milk powder room temperature closed $2 \mathrm{~h}$, add a good dilution of a resistance, 4 degrees overnight, TBST wash 2 times, each time $10 \mathrm{~min}$; add a good dilution of two anti, room temperature incubation 1 TBST, H wash 3 times, each time $10 \mathrm{~min}$. Chemiluminescence, developing and fixing. Gel image analysis. GAPDH was used as reference in this study.

\section{Statistical methods}

This study used SPSS 19.0 soft (SPSS Inc., Chicago,USA), all data are expressed as the mean \pm standard deviation (SD) val-
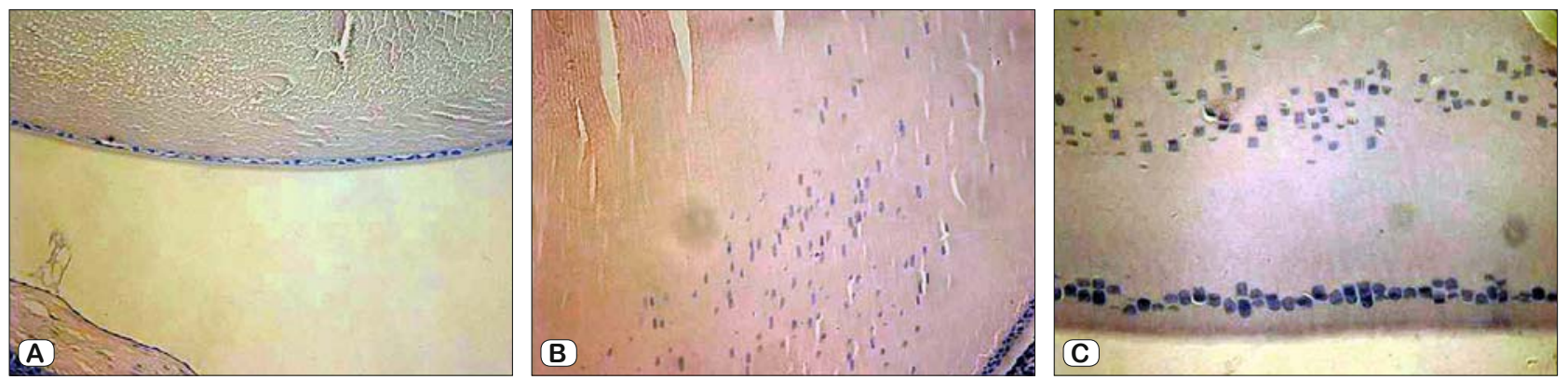

Fig. 1. HE staining, A: 0 d; B: 7 d; C: 14 d. 


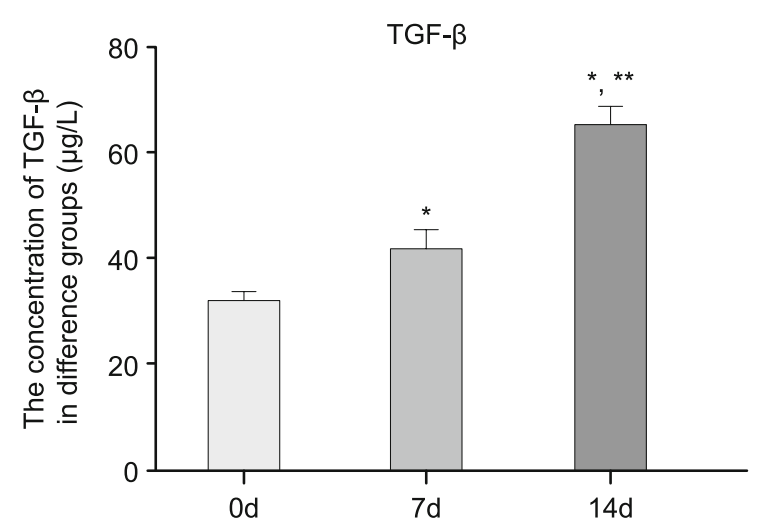

Fig. 2. The concentration of TGF- $\beta$ in different groups (ug/L), *: $p<$ 0.05 , compared with $0 \mathrm{~d}$ group, $* *: \mathrm{p}<0.05$, compared with $7 \mathrm{~d}$ group.

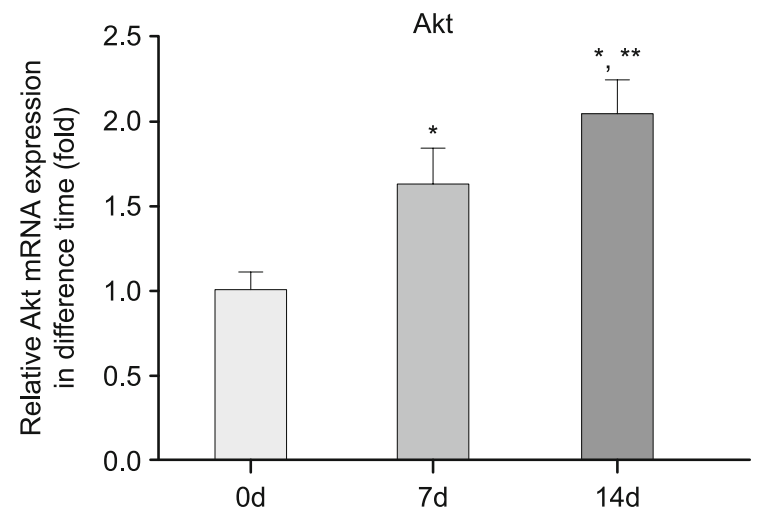

Fig. 3. Akt gene expression in different groups (Fold). *: $p<0.05$, compared with $0 d$ group, ${ }^{* *}: \mathrm{p}<0.05$, compared with $7 \mathrm{~d}$ group.

ues $(\mathrm{x} \pm \mathrm{s})$. Using one way ANOVA with LSD analysis methods in differences groups, $\mathrm{p}<0.0$ considered as statistically significant.

\section{Results}

\section{HE staining}

The lens epithelial cells located in the anterior capsule and equator capsule, simple cuboidal epithelial cells, cell shape, uniform distribution of cells from the crystal capsule wrapped, no lens fiber cell core and at the center of the abnormal (Fig. 1A). The anterior surface of the lens fiber covers the lens epithelial cells, and a large number of non degradable nuclei are seen in the lens fiber cells of the posterior part of the equator to the posterior capsule in $7 \mathrm{~d}$ and $14 \mathrm{~d}$ with time expanding (Figs 1B and 1C).

TGF-beta concentration detected in aqueous humor by Elisa method

Postoperative day 0,7 and 14 after operation in aqueous humor of TGF- $\beta$ expression respectively $(31.94+2.05) \mathrm{ug} / \mathrm{L},(41.92+$ $3.86) \mathrm{ug} / \mathrm{L}$ and $(65.65+3.35) \mathrm{ug} / \mathrm{L}$, with the passage of time, real

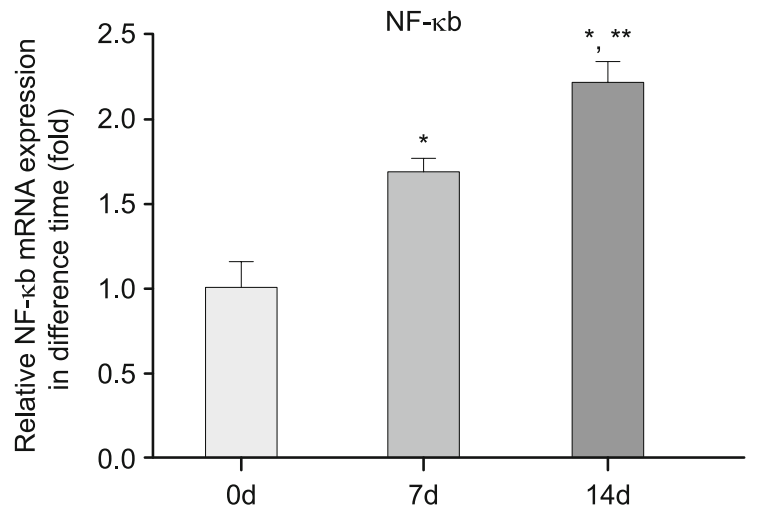

Fig. 4. NF-кb gene expression in different groups (Fold). *: $p<0.05$, compared with 0d group, ${ }^{* *}: \mathrm{p}<0.05$, compared with $7 \mathrm{~d}$ group.

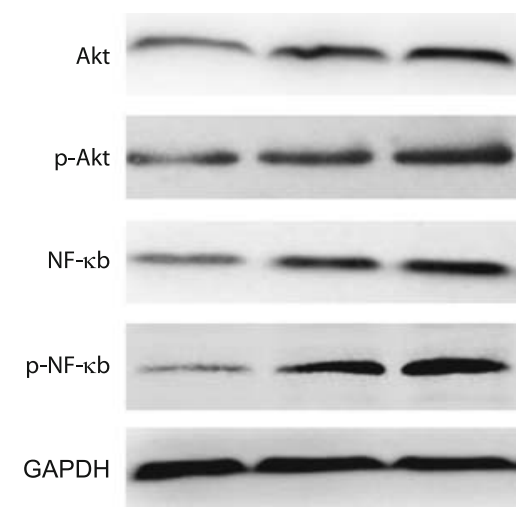

Fig. 5. Relative protein in different groups.

water TGF- $\beta$ content gradually increased, the differences between the three groups were significant $(\mathrm{P}<0.05$, respectively). The data are shown in Figure 2.

\section{RT-PCR test}

The expression levels of different time golds strong posterior Akt and NF kappa B gene, and 0d group compared to day 7 and day 14 , Akt and NF- $\kappa b$ gene expression level increased significantly ( $\mathrm{p}<0.05$, respectively); 7 and 14 days between the two groups, Akt and NF- $\kappa b$ difference was significant ( $p<0.05$, respectively). The data are shown in Figures 3 and 4.

\section{Western Blot test}

With the postoperative time, Akt and NF- $\mathrm{kb}$ protein expression levels were significantly increased and postoperative days 7 and 14 of Akt, p-Akt, NF-kb and p-NF-kb protein expressions were significantly higher than that of days 0,7 and 14 between the two groups, Akt and NF-kb differences were significant. The data are shown in Figure 5. 


\section{Discussion}

After cataract extraction (including phacoemulsification) or lens injury, residual cortex or LEC hyperplasia, the formation of turbidity, known as PCO. Some previous studies have found that cataract surgery destroyed the blood ocular barrier and various cytokines from the blood into real water, the precise and orderly LEC normal differentiation program is disrupted, large amounts of cytokines stimulation residues in the anterior capsule and equatorial portion of LEC. The proliferation, migration, and EMT and collagen fibers in the extracellular matrix of the deposition of large amounts, resulting in posterior capsular wrinkle, shrink and turbidity. At present, many studies have confirmed that the abnormal deposition of LEC and extracellular matrix induced by TGF- beta is the key to the formation of $\operatorname{PCO}(7,8)$.

A variety of signaling pathways are involved in the occurrence and development of PCO (9-11). Akt/NF-kb signaling pathway is a common signal transduction pathway in eukaryotic cells. It is involved in cell polarity, cell division, proliferation and apoptosis, cell adhesion and migration, and so on $(12,13)$. Related studies confirmed that the inhibition of TGF- beta upstream protein can effectively inhibit the collagen gel contraction induced by TGFand the expression of type I collagen $(14,15)$.

This study found that through the establishment of animal model of SD rat PCO, aqueous TGF beta content increased, posterior capsular Akt, p-Akt, NF kappa B and p-NF-kappa B, expression increased, Akt/NF- kappa B signaling pathway is activated so as to promote the proliferation of LEC and the lens transparency effect. Previous studies have confirmed that TGF-beta can regulate the related genes and proteins by activating the RhoA signaling pathway, which can induce the production of tension fibers and EMT (16-18). Studies had confirmed that Akt/NF-nuclear factor kappa $\mathrm{B}$ can induce the formation of TGF- $\beta(19,20)$, However, it is not clearly that Akt/NF-kappa B signaling pathway has a role in PCO.

The results of this study showed that TGF- $\beta$ was activated in the aqueous humor after cataract surgery in rats. The concentration of $0 \mathrm{~d}$ was gradually increased with the passage of time, and the concentration of 0dTGF- was the lowest, and $14 \mathrm{~d}$ reached the peak after operation. We found that the expression of $0 \mathrm{~d}$ was the lowest, and then gradually increased, and $14 \mathrm{~d}$ reached the peak after operation depend on respectively measuring gene expression of Akt and NF-kb and protein expression of Akt, p-Akt, NF-kb and $\mathrm{p}-\mathrm{NF}-\mathrm{kb}$ by RT-PCR and Western blot methods. According to the experimental results, we can see that the postoperative rats of posterior lens capsule on Akt and NF kappa B expression and at the same time, the main rooms water TGF beta expression was consistent, showing a time dependent trend, which can be inferred in rats after cataract surgery in aqueous humor of TGF beta is activated, following the activation of kappa Akt/NF- B signal pathway and induced EMT of lens, which promote the development of PCO.

\section{References}

1. Sundelin K, Sjostrand J. Posterior capsule opacification 5 years after extracapsular cataract extraction. J Cataract Refract Surg 1999; 25 (2): 246-250.
2. Findl O, Buehl W, Bauer $\mathbf{P}$ et al. Interventions for preventing posterior capsule opacification. Cochrane Database Syst Rev 2010; (2): D3738.

3. Lee EH, Joo CK. Role of transforming growth factor-beta in transdifferentiation and fibrosis of lens epithelial cells. Invest Ophthalmol Vis Sci 1999; 40 (9): 2025-2032.

4. Yao K, Ye PP, Tan J et al. Involvement of PI3K/Akt pathway in TGFbeta2-mediated epithelial mesenchymal transition in human lens epithelial cells. Opthalmic Res 2008; 40 (2): 69-76.

5. Tsapara A, Luthert $\mathbf{P}$, Greenwood J et al. The RhoA activator GEF-H1/ $\mathrm{Lfc}$ is a transforming growth factor-beta target gene and effector that regulates alpha-smooth muscle actin expression and cell migration. Mol Biol Cell 2010; 21 (6): 860-870.

6. Komatsu Y, Ibi M, Chosa $\mathrm{N}$ et al. Zoledronic acid suppresses transforming growth factor- $\beta$-induced fibrogenesis by human gingival fibroblasts. Int J Mol Med 2016. doi: 10.3892/ijmm.

7. Ma B, Kang Q, Qin L et al. TGF- $\beta 2$ induces transdifferentiation and fibrosis in human lens epithelial cells via regulating gremlin and CTGF. Biochem Biophys Res Comm 2014; 447 (4): 689-695.

8. Chen X, Ye S, Xiao $W$ et al. TGF- $\beta 2$ induces transdifferentiation and fibrosis in human lens epithelial cells via regulating gremlin and CTGF. Int J Mol Med 2014; 33 (6): 1664-1670.

9. Dawes LJ, Sleeman MA, Anderson IK et al. TGFbeta/Smad4-dependent and -independent regulation of human lens epithelial cells. 2009; 5 (11): 5318-5327.

10. Kayastha F, Johar K, Gaiiar D et al. Andrographolide suppresses epithelial mesenchymal transition by inhibition of MAPK signalling pathway inlens epithelial cells. J Biosci 2015; 40 (2): 313-324.

11. Park JY, Kang KA, Kim KC et al. Morin Induces Heme Oxygenase-1 via ERK-Nrf2 Signaling Pathway. J Cancer Prev 2013; 18 (3): 249-256.

12. Li X, Chen F, Zhu Q et al. Gli-1/PI3K/AKT/NF-kB pathway mediates resistance to radiation and is a target for reversion of responses in refractory acute myeloid leukemia cells. Oncotarget 2016. doi: 10.18632/oncotarget.8844.

13. Chiu CT, Chen JH, Chou FP et al. Hibiscus sabdariffa Leaf Extract Inhibits Human Prostate Cancer Cell Invasion via Down-Regulation of Akt/ NF-kB/MMP-9 Pathway. 2015; 7 (7): 5065-5087.

14. Xu P, Zhang Y, Liu Y et al. Fibroblast growth factor 21 attenuates hepatic fibrogenesis through $\mathrm{TGF}-\beta / \mathrm{smad} 2 / 3$ and $\mathrm{NF}-\kappa \mathrm{B}$ signaling pathways. Toxicol Appl Pharmacol 2016; 290: 43-53.

15. Itoh Y, Kimoto K, Imaizumi $M$ et al. Inhibition of RhoA/Rho- kinase pathway suppresses pathway the expression of type I collagen induced by TGF-beta2 inhuman retinal pigment epithelial cells. Exp Eye Res 2007; 84 (3): 464-472.

16. Xiang S, Li M, Xie X et al. Rapamycin inhibits Epithelial-to-Mesenchymal Transition of Peritoneal Mesothelium Cells through Regulation of RhoGTPases. FEBS J 2015. doi: 10.1111/febs.

17. Saika S. Relationship between posterior capsule opacification and intraocullar lens biocompatibility. Prog Retin Eye Res 2004; 23 (3): 283-305.

18. Sun CB, Teng WQ, Cui JT et al. The effect of anti-TGF- $\beta 2$ antibody functionalized intraocular lens on lens epithelial celll migration and epithelial-mesenchymal transition. Colloids Surf B Bionterfaces 2014; 113: 33-42.

19. Huang C, Wan L, Liu J. Effect of Xinfeng capsule on nuclear factor Kappa B/tumor necrosis factor alpha and transforming growth factor beta 1 / Smads pathways inrats with cardiac injuries induced by adjuvant arthritis. J Tradit Chin Med 2016; 36 (1): 92-100.

20. Tian B, Li X, Kalita M et al. Analysis of the TGF $\beta$-induced program in primary airway epithelial cells shows essential role of $N F-\kappa B / R e l A$ signaling network in type II epithelial mesenchymal transition. BMC Genomics 2015. doi: 10.1186/s12864-015-1707-x.

Received February 13, 2017. Accepted April 10, 2017. 